\title{
Comparing Expressibility of Normed BPA and Normed BPP Processes.
}

\author{
Ivana Černá \\ Mojmír Křetínský \\ Antonín Kučera \\ cerna@fi.muni.cz \\ mojmir@fi.muni.cz \\ tony@fi.muni.cz
Faculty of Informatics, Masaryk University
Botanická 68a, 60200 Brno
Czech Republic

\begin{abstract}
We compare the classes of behaviours (transition systems) which can be generated by normed $\mathrm{BPA}_{\tau}$ and normed $\mathrm{BPP}_{\tau}$ processes. We exactly classify the intersection of these two classes, i.e. the class of transition systems which can be equivalently (up to bisimilarity) described by the syntax of normed $\mathrm{BPA}_{\tau}$ and normed $\mathrm{BPP}_{\tau}$ processes. We provide such a characterisation for classes of normed BPA and normed BPP processes as well.

Next we show that it is decidable in polynomial time whether for a given normed $\mathrm{BPA}_{\tau}$ (or $\mathrm{BPP}_{\tau}$ respectively) process $\Delta$ there is some (unspecified) normed $\mathrm{BPP}_{\tau}$ (or $\mathrm{BPA}_{\tau}$ respectively) process $\Delta^{\prime}$ such that $\Delta$ is bisimilar to $\Delta^{\prime}$. Moreover, if the answer is positive then our algorithms also construct the process $\Delta^{\prime}$. Simplified versions of the algorithms mentioned above for normed BPA and normed BPP are given too.

As an immediate (but important) consequence we also obtain the decidability of bisimilarity in the union of normed $\mathrm{BPA}_{\tau}$ and normed $\mathrm{BPP}_{\tau}$ processes.
\end{abstract}

\section{Introduction}

We study a relationship between the classes of transition systems, which are generated by normed $\mathrm{BPA}_{\tau}[\mathrm{BK} 88]$ and normed $\mathrm{BPP}_{\tau}$ [Chr93] processes. We also examine such a relationship between their respective subclasses, namely normed BPA and normed BPP processes. BPA processes form type 2 class of prefix processes in Chomsky hierarchy for processes as given in [Sti96] and [Mol96], whereas BPP form type 2 class of commutative processes in this hierarchy.

BPA processes can be seen as simple sequential programs (they are equipped with a binary sequential operator). This class of processes has been intensively studied by many researchers. Baeten, Bergstra and Klop proved in [BBK87] that bisimilarity is decidable for normed BPA processes. Much simpler proofs of this were later given in [Cau88], [HS91] 
and [Gro91]. In [HS91] Hüttel and Stirling used a tableau decision method and gave also sound and complete equational theory. Hirshfeld, Jerrum and Moller demonstrated in [HJM94a] that the problem is decidable in polynomial time. The decidability result was later extended to the whole class of BPA processes by Christensen, Hüttel and Stirling in [CHS92].

If we replace the binary sequential operator with the parallel operator, we obtain BPP processes. They can thus be seen as simple parallel programs. Christensen, Hirshfeld and Moller proved in [CHM93] that bisimilarity is decidable for BPP processes. A polynomial decision algorithm for normed BPP processes was presented in [HJM94b] by Hirshfeld, Jerrum and Moller.

If we allow a parallel operator not to specify just merge but also an internal communication between two BPP processes resulting in a special action $\tau$, we obtain the class of $\mathrm{BPP}_{\tau}$ processes $([\mathrm{Chr} 93])$. In order to compare this class with its sequential counterpart we employ the class of $\mathrm{BPA}_{\tau}$ processes $([\mathrm{BK} 88])$. Decidability and complexity results just mentioned hold for these classes as well.

An interesting problem is, what is the exact relationships between $\mathrm{BPA}_{\tau}$ and $\mathrm{BPP}_{\tau}$ processes and that between their subclasses BPA and BPP, i.e. what is the relationship between sequencing and parallelism (possibly allowing simple communication). We answer these questions for normed subclasses of processes just mentioned. In the sequel we denote these subclasses by $\mathrm{nBPA}_{\tau}, \mathrm{nBPA}, \mathrm{nBPP}_{\tau}$ and $\mathrm{nBPP}$ respectively.

Our paper is organised as follows. First we recall some basic definitions and properties of $\mathrm{BPA}_{\tau}, \mathrm{BPP}_{\tau}$ and regular processes which are relevant to the subject of our paper. In Section 3 we give an exact characterisation of those transition systems which can be equivalently (up to bisimilarity) described by the syntax of $\mathrm{nBPP}_{\tau}$ and $\mathrm{nBPA}_{\tau}$ processes. Next we show that if we restrict ourselves to nBPA and nBPP processes we obtain much simpler (and hopefully nice) characterisation of those behaviours which are common to these subclasses. In Section 4 we demonstrate it is decidable whether for a given nBPA $\left(\mathrm{nBPA}_{\tau}, \mathrm{nBPP}_{\tau}, \mathrm{nBPP}\right.$ respectively) process $\Delta$ there is some (unspecified) $\mathrm{nBPP}\left(\mathrm{nBPP}_{\tau}\right.$, $\mathrm{nBPA}_{\tau}, \mathrm{nBPA}$ respectively) process $\Delta^{\prime}$ such that $\Delta \sim \Delta^{\prime}$. These algorithms are polynomial. We also show that if the answer to the previous question is positive, then the process $\Delta^{\prime}$ can be effectively constructed. Hence, as an important consequence we also obtain the decidability of bisimulation equivalence in the union of normed $\mathrm{BPA}_{\tau}$ and normed $\mathrm{BPP}_{\tau}$ processes. We conclude with remarks to related works and future research.

In many constructions of our paper we use the fact that regularity is decidable for normed BPA and normed BPP processes in polynomial time (a process is regular if it is bisimilar to a process with finitely many states). Regularity of BPA processes was examined for the first time by Mauw and Mulder in [MM94], but their notion of regularity is different from the usual one. Kučera showed in [Kuč95] that the result of Mauw and Mulder can be used to decide regularity of normed BPA processes (BPA ${ }_{\tau}$ case is an easy consequence) and that regularity of normed $\mathrm{BPP}$ and normed $\mathrm{BPP}_{\tau}$ processes is also decidable. These algorithms are polynomial. 


\section{Basic definitions, preliminary knowledge}

\section{$2.1 \quad \mathrm{BPA}_{\tau}$ and $\mathrm{BPP}_{\tau}$ processes}

Let $\Lambda=\{a, b, c, \ldots\}$ be a countably infinite set of atomic actions such that for each $a \in \Lambda$ there is a corresponding dual action $\bar{a}$ with the convention that $\overline{\bar{a}}=a$. Let $A c t=\Lambda \cup\{\tau\}$ where $\tau \notin \Lambda$ is a special (silent) action. Let $\operatorname{Var}=\{X, Y, Z, \ldots\}$ be a countably infinite set of variables such that $\operatorname{Var} \cap$ Act $=\emptyset$. The classes of recursive $\mathrm{BPA}_{\tau}$ and $\mathrm{BPP}_{\tau}$ expressions are defined by the following abstract syntax equations:

$$
\begin{array}{ll}
E_{B P A \tau} & ::=a-X-E_{B P A_{\tau}} \cdot E_{B P A_{\tau}}-E_{B P A_{\tau}}+E_{B P A_{\tau}} \\
E_{B P P_{\tau}} & ::=a-X-a E_{B P P_{\tau}}-E_{B P P_{\tau}} \mid E_{B P P_{\tau}}-E_{B P P_{\tau}}+E_{B P P_{\tau}}
\end{array}
$$

Here $a$ ranges over Act (note that we allow also $\tau$ as an action prefix) and $X$ ranges over Var. The symbol Act* denotes the set of all finite strings over Act.

As usual, we restrict our attention to guarded expressions. $\mathrm{A} \mathrm{BPA}_{\tau}$ or $\mathrm{BPP}_{\tau}$ expression $E$ is guarded if every variable occurrence in $E$ is within the scope of an atomic action.

A guarded $B P A_{\tau}$ (or $B P P_{\tau}$ ) process is defined by a finite family $\Delta$ of recursive process equations

$$
\Delta=\left\{X_{i} \stackrel{\text { def }}{=} E_{i} \quad \mid \quad 1 \leq i \leq n\right\}
$$

where $X_{i}$ are distinct elements of Var and $E_{i}$ are guarded $\mathrm{BPA}_{\tau}$ (or $\mathrm{BPP}_{\tau}$ ) expressions, containing variables from $\left\{X_{1}, \ldots, X_{n}\right\}$. The set of variables which appear in $\Delta$ is denoted by $\operatorname{Var}(\Delta)$.

The variable $X_{1}$ plays a special role $\left(X_{1}\right.$ is sometimes called the leading variable - it is a root of a labelled transition system, defined by the process $\Delta$ and following rules:

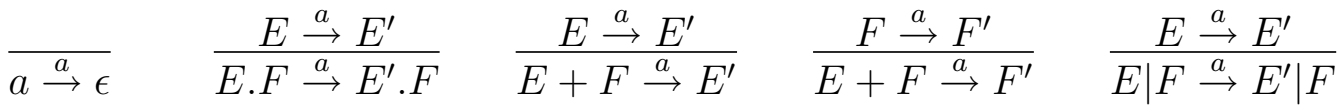

$$
\begin{aligned}
& \frac{F \stackrel{a}{\rightarrow} F^{\prime}}{E|F \stackrel{a}{\rightarrow} E| F^{\prime}} \quad \frac{E \stackrel{a}{\rightarrow} E^{\prime} \quad F \stackrel{\bar{a}}{\rightarrow} F^{\prime}}{E\left|F \stackrel{\tau}{\rightarrow} E^{\prime}\right| F^{\prime}}(a \neq \tau) \quad \frac{E \stackrel{a}{\rightarrow} E^{\prime}}{X \stackrel{a}{\rightarrow} E^{\prime}}(X \stackrel{\text { def }}{=} E \in \Delta)
\end{aligned}
$$

The symbol $\epsilon$ denotes the empty expression with usual conventions: $\epsilon|E=E, E| \epsilon=E$ and $\epsilon . E=E$. Nodes of the transition system generated by $\Delta$ are $\mathrm{BPA}_{\tau}\left(\right.$ or $\left.\mathrm{BPP}_{\tau}\right)$ expressions, which are often called states of $\Delta$, or just "states" when $\Delta$ is understood from the context. We also define the relation $\stackrel{w}{\rightarrow}^{*}$, where $w \in A c t^{*}$, as the reflexive and transitive closure of $\stackrel{a}{\rightarrow}$ (we often write $E \rightarrow^{*} F$ instead of $E \stackrel{w}{\rightarrow}^{*} F$ if $w$ is irrelevant). Given two states $E, F$, we say that $F$ is reachable from $E$, if $E \rightarrow^{*} F$. States of $\Delta$ which are reachable from $X_{1}$ are said to be reachable.

Remark 1. Processes are often identified with their leading variables. Furthermore, if we assume a fixed process $\Delta$, we can view any process expression $E$ (not necessarily guarded) whose variables are defined in $\Delta$ as a process too; we simply add a new leading equation $X \stackrel{\text { def }}{=} E^{\prime}$ to $\Delta$, where $X$ is a variable from $\operatorname{Var}$ such that $X \notin \operatorname{Var}(\Delta)$ and $E^{\prime}$ is a process expression which is obtained from $E$ by substituting each variable in $E$ with the right-hand 
side of its corresponding defining equation in $\Delta$ ( $E^{\prime}$ must be guarded now). All notions originally defined for processes can be used for process expressions in this sense too.

\subsubsection{Bisimulation}

The equivalence between process expressions (states) we are interested in here is bisimilarity [Par81], defined as follows:

Definition 1. A binary relation $R$ over process expressions is a bisimulation if whenever $(E, F) \in R$ then for each $a \in$ Act

- if $E \stackrel{a}{\rightarrow} E^{\prime}$, then $F \stackrel{a}{\rightarrow} F^{\prime}$ for some $F^{\prime}$ such that $\left(E^{\prime}, F^{\prime}\right) \in R$

- if $F \stackrel{a}{\rightarrow} F^{\prime}$, then $E \stackrel{a}{\rightarrow} E^{\prime}$ for some $E^{\prime}$ such that $\left(E^{\prime}, F^{\prime}\right) \in R$

Processes $\Delta$ and $\Delta^{\prime}$ are bisimilar, written $\Delta \sim \Delta^{\prime}$, if their leading variables are related by some bisimulation.

\subsubsection{Normed processes}

An important subclass of $\mathrm{BPA}_{\tau}$ and $\mathrm{BPP}_{\tau}$ processes can be obtained by an extra restriction of normedness. A variable $X \in \operatorname{Var}(\Delta)$ is normed if there is $w \in A c t^{*}$ such that $X \stackrel{w}{\rightarrow} \epsilon$. In that case we define the norm of $X$, written $|X|$, to be the length of the shortest such $w$. In case of $\mathrm{BPP}_{\tau}$ processes we also require that no $\tau$ action which appears in $w$ is a result of a communication on dual actions in the sense of operational semantics given above. This is necessary if we want the norm to be additive over '|' operator ( $\tau$ can still occur in $w$-remember it can be used as an action prefix). A process $\Delta$ is normed if all variables of $\operatorname{Var}(\Delta)$ are normed. The norm of $\Delta$ is then defined to be the norm of $X_{1}$. Note the norm of a normed process is easy to compute and bisimilar processes must have the same norm.

\subsubsection{Greibach normal form}

Any $\mathrm{BPA}_{\tau}$ or $\mathrm{BPP}_{\tau}$ process $\Delta$ can be effectively presented in so-called 3-Greibach normal form (see [BBK87] and [Chr93]). Before the definition we need to introduce the set $\operatorname{Var}(\Delta)^{*}$ of all finite sequences of variables from $\operatorname{Var}(\Delta)$, and the set $\operatorname{Var}(\Delta)^{\otimes}$ of all finite multisets over $\operatorname{Var}(\Delta)$. Each multiset of $\operatorname{Var}(\Delta)^{\otimes}$ denotes a $\mathrm{BPP}_{\tau}$ expression by combining its elements in parallel using the '|' operator.

Definition 2. $A B P A_{\tau}$ (or $B P P_{\tau}$ ) process $\Delta$ is said to be in Greibach normal form (GNF) if all its equations are of the form

$$
X \stackrel{\text { def }}{=} \sum_{j=1}^{n} a_{j} \alpha_{j}
$$

where $n \in N, a_{j} \in$ Act and $\alpha_{j} \in \operatorname{Var}(\Delta)^{*}$ (or $\alpha_{j} \in \operatorname{Var}(\Delta)^{\otimes}$ ). If Length $\left(\alpha_{j}\right) \leq 2$ (or $\left.\operatorname{card}\left(\alpha_{j}\right) \leq 2\right)$ for each $j, 1 \leq j \leq n$, then $\Delta$ is said to be in 3-GNF. 
From now on we assume that all $\mathrm{BPA}_{\tau}$ and $\mathrm{BPP}_{\tau}$ processes we are working with are presented in GNF. This justifies also the assumption that all reachable states of a $\mathrm{BPA}_{\tau}$ process $\Delta$ are elements of $\operatorname{Var}(\Delta)^{*}$ and all reachable states of a $\mathrm{BPP}_{\tau}$ process $\Delta^{\prime}$ are elements of $\operatorname{Var}\left(\Delta^{\prime}\right)^{\otimes}$.

Remark 2. In the rest of this paper we let Greek letters $\alpha, \beta, \ldots$ range over reachable states of a $B P A_{\tau}$ or $B P P_{\tau}$ process $\triangle$ in GNF. Occasionally we also use the notation $\alpha^{i}$ with the following meaning:

$$
\begin{aligned}
& \alpha^{i}=\underbrace{\alpha . \alpha \cdots \alpha}_{i} \text { if } \alpha \text { is a state of some BPA } A_{\tau} \text { process in GNF } \\
& \alpha^{i}=\underbrace{\alpha|\alpha \cdots| \alpha}_{i} \text { if } \alpha \text { is a state of some BPP } P_{\tau} \text { process in } G N F
\end{aligned}
$$

\subsection{Regular processes}

Many proofs in this paper take advantage of the fact that regularity of normed $\mathrm{BPA}_{\tau}$ and normed $\mathrm{BPP}_{\tau}$ processes is decidable in polynomial time. Regularity of BPA processes was examined for the first time by Mauw and Mulder in [MM94], but their notion of regularity is different from the usual one. Kučera showed in [Kuč95] that the result of Mauw and Mulder can be used to decide regularity of normed BPA (and thus also BPA ${ }_{\tau}$ ) processes and that regularity of normed $\mathrm{BPP}$ and $\mathrm{BPP}_{\tau}$ processes is also decidable. These algorithms are polynomial. The next definition explains what is meant by the notion of regularity and introduce standard normal form for regular processes.

Definition 3. A process $\Delta$ is regular if there is a process $\Delta^{\prime}$ with finitely many states such that $\Delta \sim \Delta^{\prime}$. A regular process $\Delta$ is said to be in normal form if all its equations are of the form

$$
X \stackrel{\text { def }}{=} \sum_{j=1}^{n} a_{j} X_{j}
$$

where $n \in N, a_{j} \in$ Act and $X_{j} \in \operatorname{Var}(\Delta)$.

It is easy to see that a process is regular iff it can reach only finitely many states up to bisimilarity. In [Mil89] it is shown, that regular processes can be represented in the normal form just defined. Thus a process $\Delta$ is regular iff there is a regular process $\Delta^{\prime}$ in normal form such that $\Delta \sim \Delta^{\prime}$. Now we present several propositions which concern regularity of normed $\mathrm{BPA}_{\tau}$ and normed $\mathrm{BPP}_{\tau}$ processes. Proofs can be found in [Kuč95].

Proposition 1. Let $\Delta$ be a normed $B P A_{\tau}$ or $B P P_{\tau}$ process. The problem whether $\Delta$ is regular is decidable in polynomial time. Moreover, if $\Delta$ is regular then a regular process $\Delta^{\prime}$ in normal form such that $\Delta \sim \Delta^{\prime}$ can be effectively constructed.

Definition 4. Let $\Delta$ be a normed $B P A_{\tau}$ (or $B P P_{\tau}$ ) process. A variable $Y \in \operatorname{Var}(\Delta)$ is growing if $Y \rightarrow^{*} Y$. $\alpha$ (or $Y \rightarrow^{*} Y \mid \alpha$ ) where $\alpha \in \operatorname{Var}(\Delta)^{*}$ such that Length $(\alpha) \geq 1$ (or $\alpha \in \operatorname{Var}(\Delta)^{\otimes}$ such that $\left.\operatorname{card}(\alpha) \geq 1\right)$. 
Proposition 2. A normed $B P A_{\tau}$ (or $B P P_{\tau}$ ) process $\Delta$ is non-regular iff $\operatorname{Var}(\Delta)$ contains a growing variable $Y$ such that there is a reachable state of the form $Y$. $\beta$ where $\beta \in \operatorname{Var}(\Delta)^{*}$ (or the state $Y$ is reachable).

Remark 3. If $\Delta$ is a normed $B P A_{\tau}$ (or $B P P_{\tau}$ ) process and $\alpha$ is a $B P A_{\tau}$ (or $B P P_{\tau}$ ) expression whose variables are defined in $\Delta$, then Proposition 2 can be applied also to $\alpha$ - each such expression denotes a process in the sense of Remark 1. Namely variables of $\triangle$ are $B P A_{\tau}$ and $B P P_{\tau}$ expressions - hence we can also speak about regular variables.

\section{The characterisation of $\mathbf{n B P A}_{\tau} \cap \mathbf{n B P P} \mathbf{P}_{\tau}$}

In this section we give an exact characterisation of those normed processes which can be equivalently defined by both $\mathrm{BPA}_{\tau}$ and $\mathrm{BPP}_{\tau}$ syntax.

Definition 5 (the class $\mathrm{nBPA}_{\tau} \cap \mathrm{nBPP}_{\tau}$ ). Let $n B P A_{\tau}$ and $n B P P_{\tau}$ denote the classes of normed $B P A_{\tau}$ and normed $B P P_{\tau}$ processes, respectively. We define the class $n B P A_{\tau} \cap n B P P_{\tau}$ in the following way:

$$
\begin{aligned}
n B P A_{\tau} \cap n B P P_{\tau}= & \left\{\Delta \in n B P A_{\tau}, \quad \mid \exists \Delta^{\prime} \in n B P P_{\tau} \text { such that } \Delta \sim \Delta^{\prime}\right\} \cup \\
& \left\{\Delta \in n B P P_{\tau}, \mid \exists \Delta^{\prime} \in n B P A_{\tau} \text { such that } \Delta \sim \Delta^{\prime}\right\}
\end{aligned}
$$

The class $\mathrm{nBPA}_{\tau} \cap \mathrm{nBPP}_{\tau}$ can be seen as a "semantical intersection" of $\mathrm{nBPA}_{\tau}$ and $\mathrm{nBPP}_{\tau}$. It is clearly nonempty because each normed finite-state process belongs to $\mathrm{nBPA}_{\tau} \cap \mathrm{nBPP}_{\tau}$. But $n B P A_{\tau} \cap n P_{\tau}$ contains also processes with infinitely many states - assume the following process:

$$
X \stackrel{\text { def }}{=} a(X \mid X)+a
$$

$X$ is a normed $\mathrm{BPP}_{\tau}$ process with infinitely many states. If we replace the operator 'I' with '.', we obtain a bisimilar $\mathrm{BPA}_{\tau}$ process:

$$
\bar{X} \stackrel{\text { def }}{=} a(\bar{X} \cdot \bar{X})+a
$$

Clearly $X \sim \bar{X}$ because transition systems generated by those processes are even isomorphic:

$$
0<\frac{a}{<} \bullet \stackrel{a}{\underset{a}{\longleftrightarrow}} 0 \stackrel{a}{\underset{a}{\longleftrightarrow}} \mathrm{O} \underset{a}{\stackrel{a}{\longleftarrow}} \mathrm{O}
$$

Now we modify the process $X$ slightly:

$$
X \stackrel{\text { def }}{=} a(X \mid X)+a+\bar{a}
$$

Although the process (3) does not differ from the process (1) too much, it is not hard to prove that there is no $\mathrm{BPA}_{\tau}$ process bisimilar to (3).

In [ČKK96] it is proved that each normed $\mathrm{BPP}_{\tau}$ and $\mathrm{BPA}_{\tau}$ process from $\mathrm{nBPA}_{\tau} \cap \mathrm{nBPP}_{\tau}$ can be represented in a special normal form denoted $\mathrm{INF}_{\mathrm{BPP}}$ and $\mathrm{INF}_{\mathrm{BPA}}$, respectively. Before the definitions of $\mathrm{INF}_{\mathrm{BPP}}$ and $\mathrm{INF}_{\mathrm{BPA}}$ we first introduce the notion of reduced processes: 
Definition 6 (reduced processes). Let $\Delta$ be a normed $B P A_{\tau}$ (or $B P P_{\tau}$ ) process in $G N F$. We say that $\Delta$ is reduced if

1. variables of $\operatorname{Var}(\Delta)$ are pairwise non-bisimilar

2. for each $V \in \operatorname{Var}(\Delta)$ there is a reachable state of the form $\operatorname{V.\alpha }$, where $\alpha \in \operatorname{Var}(\Delta)^{*}$ (or the state $V$ is reachable).

As bisimilarity is decidable for normed $\mathrm{BPA}_{\tau}$ and normed $\mathrm{BPP}_{\tau}$ processes in polynomial time (see [HJM94a], [HJM94b]), the first condition can be assumed w.l.o.g. Variables which do not fulfil the second condition cannot contribute to the behaviour of $\Delta$ and they can be effectively removed in polynomial time. Hence we can assume (w.l.o.g.) that a normed $\mathrm{BPA}_{\tau}$ or $\mathrm{BPP}_{\tau}$ process $\Delta$ is reduced.

Definition 7 ( $\left.\mathrm{INF}_{\mathrm{BPP}}\right)$. Let $\Delta$ be a normed reduced BPP $\tau$ process in GNF.

1. A variable $Z \in \operatorname{Var}(\Delta)$ is simple if all summands in the def. equation for $Z$ are of the form $a Z^{i}$, where $a \in$ Act and $i \in N \cup\{0\}$. Moreover, at least one of those summands must be of the form $a Z^{k}$ where $a \in$ Act and $k \geq 2$. Finally, the def. equation for $Z$ must not contain two summands of the form $b, \bar{b}$, where $b \in$ Act.

2. The process $\Delta$ is said to be in $I N F_{B P P}$ if whenever a $\alpha$ is a summand in a def. equation from $\Delta$ such that Length $(\alpha) \geq 2$, then $\alpha=Z^{i}$ for some simple variable $Z$ and $i \geq 2$.

Note that if $Z$ is a simple variable, then $|Z|=1$ because $Z$ could not be normed otherwise.

Example 1. The following process as well as process (1) are in $I N F_{B P P}$, while the processes (3) is not:

$$
\begin{array}{lll}
X & \stackrel{\text { def }}{=} & a Y+b(Z \mid Z)+b+\bar{b} \\
Y & \stackrel{\text { def }}{=} & c Y+b X+a(Z|Z| Z) \\
Z & \stackrel{\text { def }}{=} & a(Z \mid Z)+\bar{a}(Z|Z| Z)+b+\bar{a}
\end{array}
$$

Remark 4. The set of all reachable states of a process $\Delta$ in $I N F_{B P P}$ looks as follows:

$$
\operatorname{Var}(\Delta) \cup\left\{Z^{i} \mid Z \in \operatorname{Var}(\Delta) \text { is a simple variable and } i \in N \cup\{0\}\right\}
$$

Proposition 3. Each process $\Delta$ in $I N F_{B P P}$ belongs to $n B P A_{\tau} \cap n B P P_{\tau}$.

Proof: We show how to construct a normed $\mathrm{BPA}_{\tau}$ process $\bar{\Delta}$, which is bisimilar to $\Delta$. First we need to define the notion of closed simple variables - a simple variable $Z \in \operatorname{Var}(\Delta)$ is closed if the following condition holds: If the def. equation for $Z$ contains two summands of the form $b Z^{i}, \bar{b} Z^{j}$, then it also contains a summand $\tau Z^{i+j-1}$ (note that $Z$ is simple, hence the case $i=j=0$ is impossible).

The set of variables of $\bar{\Delta}$ looks as follows: for each $V \in \operatorname{Var}(\Delta)$ we fix a fresh variable $\bar{V}$. Moreover, for each simple non-closed variable $Z \in \operatorname{Var}(\Delta)$ we also fix a fresh variable $\bar{Z}_{C}$. Now we can start to transform $\Delta$ into $\bar{\Delta}$. For each equation $Y \stackrel{\text { def }}{=} \sum_{i=1}^{n} a_{i} \alpha_{i}$ of $\Delta$ we add the equation $\bar{Y} \stackrel{\text { def }}{=} \sum_{i=1}^{n} a_{i} \mathcal{T}\left(\alpha_{i}\right)$ to $\bar{\Delta}$, where $\mathcal{T}$ is defined as follows: 
1. $\mathcal{T}(V)=\bar{V}$, where $V \in \operatorname{Var}(\Delta)$.

2. $\mathcal{T}\left(Z^{i}\right)=\bar{Z}^{i}$, where $i \geq 2$ and $Z \in \operatorname{Var}(\Delta)$ is a closed simple variable.

3. $\mathcal{T}\left(Z^{i}\right)=\bar{Z}_{C}^{i-1} \cdot \bar{Z}$, where $i \geq 2$ and $Z \in \operatorname{Var}(\Delta)$ is a non-closed simple variable.

The defining equation for $\bar{Z}_{C}$, where $Z \in \operatorname{Var}(\Delta)$ is a non-closed simple variable, is constructed using following rules:

1. if $a Z^{i}$ is a summand in the def. equation for $Z$, then $a \bar{Z}_{C}^{i}$ is a summand in the def. equation for $\bar{Z}_{C}$ in $\bar{\Delta}$.

2. if $b Z^{i}, \bar{b} Z^{j}$ are summands in the def. equation for $Z$, then $\tau \bar{Z}_{C}^{i+j-1}$ is a summand in the def. equation for $\bar{Z}_{C}$ in $\bar{\Delta}$.

The fact $\Delta \sim \bar{\Delta}$ is easy to check.

Example 2. If we apply the transformation algorithm to the process from Example 1, we obtain the following bisimilar $B P A_{\tau}$ process:

$$
\begin{aligned}
& \bar{X} \stackrel{\text { def }}{=} a \bar{Y}+b\left(\bar{Z}_{C} \cdot \bar{Z}\right)+b+\bar{b} \\
& \bar{Y} \stackrel{\text { def }}{=} c \bar{Y}+b \bar{X}+a\left(\bar{Z}_{C} \cdot \bar{Z}_{C} \cdot \bar{Z}\right) \\
& \bar{Z} \stackrel{\text { def }}{=} a\left(\bar{Z}_{C} \cdot \bar{Z}\right)+\bar{a}\left(\bar{Z}_{C} \cdot \bar{Z}_{C} \cdot \bar{Z}\right)+b+\bar{a} \\
& \bar{Z}_{C} \stackrel{\text { def }}{=} a\left(\bar{Z}_{C} \cdot \bar{Z}_{C}\right)+\bar{a}\left(\bar{Z}_{C} \cdot \bar{Z}_{C} \cdot \bar{Z}_{C}\right)+b+\bar{a}+\tau\left(\bar{Z}_{C} \cdot \bar{Z}_{C} \cdot \bar{Z}_{C} \cdot \bar{Z}_{C}\right)+\tau \bar{Z}_{C}
\end{aligned}
$$

As we can also prove that each normed $\mathrm{BPP}_{\tau}$ process from $\mathrm{nBPA}_{\tau} \cap \mathrm{nBPP}_{\tau}$ can be represented in $\mathrm{INF}_{\mathrm{BPP}}$ (see [CKKK96]), we obtain the following theorem:

Theorem 1. The class $n B P A_{\tau} \cap n B P P_{\tau}$ contains exactly (up to bisimilarity) normed $B P P_{\tau}$ processes in $I N F_{B P P}$.

The class $\mathrm{nBPA}_{\tau} \cap \mathrm{nBPP}_{\tau}$ can also be characterised using $\mathrm{BPA}_{\tau}$ syntax. To do this, we introduce a special normal form for normed $\mathrm{BPA}_{\tau}$ processes:

Definition $8\left(\mathrm{INF}_{\mathrm{BPA}}\right)$. Let $\Delta$ be a normed reduced $B P A_{\tau}$ process in $G N F$.

1. Let $X, Y \in \operatorname{Var}(\Delta)$ be non-regular variables. We say that $Y$ is a communication closure (C-closure) of $X$ if the following conditions hold:

- All summands in the def. equation for $X$ are either of the form a where $a \in$ Act, or $a\left(Y^{i} . X\right)$ where $a \in$ Act and $i \in N \cup\{0\}$. Moreover, at least one summand is of the form $a\left(Y^{k} . X\right)$ where $k \geq 1$.

- All summands in the def. equation for $Y$ are of the form $a Y^{i}$, where a $\in$ Act and $i \in N \cup\{0\}$. 
- $a Y^{i}$ is a summand in the def. equation for $Y$ iff one of the following conditions holds:

(a) $i=0$ and $a$ is a summand in the def. equation for $X$.

(b) $i \geq 1$ and $a\left(Y^{i-1} . X\right)$ is a summand in the def. equation for $X$.

(c) $a=\tau$ and there are two summands of the form $b \alpha_{1}, \bar{b} \alpha_{2}$ in the def. equation for $X$ such that $i=$ Length $\left(\alpha_{1}\right)+$ Length $\left(\alpha_{2}\right)-1$ (note that this condition ensures that def. equations for $X, Y$ do not contain two summands of the form $b, \bar{b})$.

2. The process $\Delta$ is said to be in $I N F_{B P A}$ if whenever a $\alpha$ is a summand in a def. equation from $\Delta$ such that Length $(\alpha) \geq 2$, then $\alpha=X^{i} . Y$ for some $i \in N$ and $X, Y \in \operatorname{Var}(\Delta)$ such that $Y$ is a $C$-closure of $X$ (note that $X, Y$ need not be different-there can be variables which are $C$-closed by themselves).

Note that if $Y$ is a C-closure of $X$, then $|Y|=|X|=1$. Another interesting property of $X$ and $Y$ is presented in the following remark.

Remark 5. It is easy to check that if $Y$ is a $C$-closure of $X$, then $Y^{i} . X \sim \bar{X}^{i+1}$ where $\bar{X}$ is a normed BPP process composed of a single variable whose def. equation is obtained from the def. equation for $X$ by substituting '.' with 'l' and replacing each occurence of $X$ and $Y$ with $\bar{X}$.

The previous remark in fact says that if $\Delta$ is a normed $\mathrm{BPA}_{\tau}$ process in $\mathrm{INF}_{\mathrm{BPA}}$, then a bisimilar normed $\mathrm{BPP}_{\tau}$ process can be constructed by an algorithm which is inverse to the algorithm presented in the proof of Proposition 3.

Theorem 2. The class $n B P A_{\tau} \cap n B P P_{\tau}$ contains exactly (up to bisimilarity) normed $B P A_{\tau}$ processes in $I N F_{B P A}$.

$\mathrm{BPA}_{\tau}$ and $\mathrm{BPP}_{\tau}$ processes are often defined in a simplified form, where ' $\tau$ ' cannot be used as an action prefix and pure merge operator ' $\|$ ', which does not allow synchronisations on dual actions, is used instead of ' $\mid$ '. These process classes are denoted BPA and BPP by convention. So far we have investigated the intersection of $\mathrm{nBPA}_{\tau}$ and $\mathrm{nBPP}_{\tau}$ processes. It was desirable to work with this unrestricted syntax, because we could also examine when it is possible to simulate "real" communications in a $\mathrm{BPP}_{\tau}$ process by a sequential $\mathrm{BPA}_{\tau}$ process. Obviously these results applies to nBPA/nBPP processes as well. But the characterisation of $\mathrm{nBPA} \cap \mathrm{nBPP}$ is much simpler and therefore we present it explicitly.

Definition 9 (INF). Let $\Delta$ be a normed reduced BPA or BPP process in GNF.

1. A variable $Z \in \operatorname{Var}(\Delta)$ is simple if all summands in the def. equation for $Z$ are of the form $a Z^{i}$, where $a \in$ Act and $i \in N \cup\{0\}$. Moreover, at least one of those summands must be of the form a $Z^{k}$ where $a \in$ Act and $k \geq 2$. 
2. The process $\Delta$ is said to be in INF if whenever a $\alpha$ is a summand in a def. equation from $\Delta$ such that Length $(\alpha) \geq 2(\operatorname{or} \operatorname{card}(\alpha) \geq 2)$, then $\alpha=Z^{i}$ for some simple variable $Z$ and $i \geq 2$.

Note that normed BPA (or BPP) processes in INF have a nice property - a bisimilar normed BPP (or BPA) process can be obtained just by replacing '.' operator with 'Il' operator (or by replacing 'Il' operator with '.' operator).

Theorem 3. The class $n B P A \cap n B P P$ contains exactly (up to bisimilarity) normed BPA (or BPP) processes in INF.

\section{Deciding whether $\Delta \in \mathbf{n B P A}_{\tau} \cap \mathbf{n B P P}_{\tau}$}

In this section we prove that the problem whether a given normed $\mathrm{BPA}_{\tau}$ or $\mathrm{BPP}_{\tau}$ process $\Delta$ belongs to $\mathrm{nBPA}_{\tau} \cap \mathrm{nBPP}_{\tau}$ is decidable in polynomial time. The technique is essentially similar in both cases - we check if each summand of each defining equation of $\Delta$ whose form is not admitted by $\mathrm{INF}_{\mathrm{BPA}}$ or $\mathrm{INF}_{\mathrm{BPP}}$ can be in principal transformed so that requirements of $\mathrm{INF}_{\mathrm{BPA}}$ or $\mathrm{INF}_{\mathrm{BPP}}$ are fulfilled. We also present simplified versions of our algorithms which work for normed BPA and BPP processes.

Next we show how to modify presented algorithms so that they become constructive. Unfortunately, these algorithms are no longer polynomial. We start with some definitions:

Definition $10\left(\mathbf{S}(\boldsymbol{\Delta}), \mathbf{R}(\boldsymbol{\Delta})\right.$ and $\mathbf{G}(\boldsymbol{\Delta})$ sets). Let $\Delta$ be a normed $B P A_{\tau}$ or $B P P_{\tau}$ process in GNF.

- the set $S(\Delta) \subseteq \operatorname{Var}(\Delta)$ is composed of all variables $V$ such that $|V|=1, V$ is nonregular and if a $\alpha$ is a summand in the defining equation for $V$ in $\Delta$, then $\alpha \sim V^{|\alpha|}$.

- the set $R(\Delta) \subseteq \operatorname{Var}(\Delta)$ contains all regular variables of $\Delta$.

- the set $G(\Delta) \subseteq \operatorname{Var}(\Delta)$ contains all growing variables of $\Delta$.

The sets $S(\Delta), R(\Delta)$ and $G(\Delta)$ can be constructed in polynomial time because bisimilarity and regularity are decidable in polynomial time for normed $\mathrm{BPA}_{\tau}$ and normed $\mathrm{BPP}_{\tau}$ processes (see [HJM94a], [HJM94b] and [Kuč95]).

If $\Delta$ is a normed $\mathrm{BPA}_{\tau}$ (or $\mathrm{BPP}_{\tau}$ ) process from $\mathrm{nBPA}_{\tau} \cap \mathrm{nBPP}_{\tau}$, then there is $\Delta^{\prime}$ in $\mathrm{INF}_{\mathrm{BPA}}$ (or $\mathrm{INF}_{\mathrm{BPP}}$ ) such that $\Delta \sim \Delta^{\prime}$. In case of normed $\mathrm{BPP}_{\tau}$ processes the set $S(\Delta)$ contains in fact variables which can be (potentially) bisimilar to simple variables of $\Delta^{\prime}$. In case of normed $\mathrm{BPA}_{\tau}$ processes the set $S(\Delta)$ contains variables which can be bisimilar to C-closures of variables from $\operatorname{Var}\left(\Delta^{\prime}\right)$.

Now we present three lemmas which help us to prove the corectness of the algorithm below (it decides whether a given normed $\mathrm{BPP}_{\tau}$ process belongs to $\mathrm{nBPA}_{\tau} \cap \mathrm{nBPP}_{\tau}$ ).

Lemma 1. Let $\Delta$ be a normed reduced $B P P_{\tau}$ process in 3-GNF and let $a(A \mid B)$ be a summand of a defining equation from $\Delta$ such that $A$ is regular and $B$ is non-regular. Then $\Delta \notin n B P A_{\tau} \cap n B P P_{\tau}$. 
Proof: Assume there is a normed $\mathrm{BPP}_{\tau}$ process $\Delta^{\prime}$ in $\mathrm{INF}_{\mathrm{BPP}}$ such that $\Delta \sim \Delta^{\prime}$. Let $n=\max \left\{|Y|, Y \in \operatorname{Var}\left(\Delta^{\prime}\right)\right\}$. As $B$ is non-regular, it can reach a state of an arbitrary norm - let $B \rightarrow^{*} \beta$ where $|\beta|>n$. Then $A \mid \beta$ is a reachable state of $\Delta$ and thus $A \mid \beta \sim \beta^{\prime}$ for some reachable state $\beta^{\prime}$ of $\Delta^{\prime}$. As $|A| \beta \mid>n$, we can conclude that $\beta^{\prime} \equiv Z^{|A| \beta \mid}$ where $Z \in \operatorname{Var}\left(\Delta^{\prime}\right)$ is a simple variable (see Remark 4). Hence $A \sim Z^{|A|}$ and as each simple variable is growing (see Definition 7 ), it contradicts regularity of $A$.

Lemma 2. Let $\Delta$ be a normed reduced $B P P_{\tau}$ process in 3-GNF, $\Delta \in n B P A_{\tau} \cap n B P P_{\tau}$. Let $a(A \mid B)$ be a summand of a defining equation from $\Delta$ such that $A$ and $B$ are non-regular. Then there is exactly one variable $Z \in S(\Delta)$ such that $A \mid B \sim Z^{|A| B \mid}$.

Proof: Let $\Delta^{\prime}$ be a normed $\mathrm{BPP}_{\tau}$ process in $\mathrm{INF}_{\mathrm{BPP}}$ such that $\Delta \sim \Delta^{\prime}$. Let $n=$ $\max \left\{|Y|, \quad Y \in \operatorname{Var}\left(\Delta^{\prime}\right)\right\}$. Using the same argument as in the proof of Lemma 1 we obtain $A \sim P^{|A|}, B \sim Q^{|B|}$ where $P, Q \in \operatorname{Var}\left(\Delta^{\prime}\right)$ are simple variables. We show that $P \equiv Q$. Let $A \rightarrow^{*} \alpha$ where $|\alpha|>n$. Then clearly $\alpha \sim P^{|\alpha|}$ and as $\alpha \mid B$ is a reachable state of $\Delta, \alpha \mid B \sim R^{|\alpha| B \mid}$ where $R \in \operatorname{Var}\left(\Delta^{\prime}\right)$ is a simple variable. To sum up, we have $\alpha\left|B \sim P^{|\alpha|}\right| Q^{|B|} \sim R^{|\alpha| B \mid}$. Hence $P \sim R \sim Q$ and thus $P \equiv R \equiv Q$ because $\Delta^{\prime}$ is reduced. As e.g. $P$ is a reachable state of $\Delta^{\prime}$, there is a reachable state $\gamma$ of $\Delta$ such that $P \sim \gamma$. As $|P|=1$, we can conclude $\gamma \equiv Z$ for some $Z \in \operatorname{Var}(\Delta)$ which clearly belongs to $S(\Delta)$. Moreover, $Z$ is unique because $\Delta$ is reduced.

Lemma 3. Let $\Delta$ be a normed $B P P_{\tau}$ process in $G N F$ and let $X \in S(\Delta)$. If the defining equation for $X$ contains two summands of the form $b, \bar{b}$, then $\Delta \notin n B P A_{\tau} \cap n B P P_{\tau}$.

Proof: Assume there is a normed $\mathrm{BPP}_{\tau}$ process $\Delta^{\prime}$ in $\mathrm{INF}_{\mathrm{BPP}}$ such that $\Delta \sim \Delta^{\prime}$. Using the same kind of argument as in the proof of Lemma 1 we obtain that $X \sim Z$ for some simple variable $Z \in \operatorname{Var}\left(\Delta^{\prime}\right)$. As the def. equation for $X$ contains two summands of the form $b, \bar{b}$ and $X \sim Z$, the def. equation for $Z$ must contain those summands too-hence $Z$ is not simple and we have a contradiction.

The promised (constructive) algorithm for normed $\mathrm{BPP}_{\tau}$ processes is presented on Figure 1. Steps which are executed only by the constructive algorithm are shaded - if we omit everything on a grey background, we obtain a non-constructive polynomial algorithm. The abbreviation "NFR $(\Delta)$ " stands for the Normal Form of the Regular process $\Delta$, which can be effectively constructed (see Proposition 1). We always assume that $\operatorname{NFR}(\Delta)$ contains fresh variables which are not contained in any other process we are working with. When the command return is executed, the algorithm halts and returns the value which follows immediately after the keyword return.

The constructive algorithm is not polynomial because the construction of NFR is not polynomial - a normed regular BPP process in 3-GNF with $n$ variables can generally reach exponentially many pairwise non-bisimilar states and each of these states requires a special variable.

Our algorithm for normed $\mathrm{BPP}_{\tau}$ processes works for pure normed $\mathrm{BPP}$ processes as well. It suffices to replace the '|' operator with ' $\|$ ' operator in our description. As there 
Algorithm: A constructive test of the membership to $\mathrm{nBPA}_{\tau} \cap \mathrm{nBPP}_{\tau}$ for normed $\mathrm{BPP}_{\tau}$ processes.

Input: $\quad$ A normed reduced $\mathrm{BPP}_{\tau}$ process $\Delta$ in $3-\mathrm{GNF}$.

Output: $\quad$ YES and a normed $\mathrm{BPP}_{\tau}$ process $\Delta^{\prime}$ in $\mathrm{INF}_{\mathrm{BPP}}$ such that $\Delta \sim \Delta^{\prime}$ if $\Delta \in \mathrm{nBPA}_{\tau} \cap \mathrm{nBPP}_{\tau}$.

NO otherwise.

1. Construct the sets $S(\Delta), R(\Delta)$ and $G(\Delta)$.

2. If there is $X \in S(\Delta)$ whose def. equation contains two summands of the form $b, \bar{b}$ then $\underline{\text { return }} \mathrm{NO}$;

3. If $G(\Delta)=\emptyset \underline{\text { then }}$

$\Delta^{\prime}:=\operatorname{NFR}(\Delta)$;

return YES and $\Delta^{\prime}$;

4. $\Delta^{\prime}:=\Delta ;$

5. for each summand of the form $a(A \mid B)$ in defining equations of $\Delta \underline{\text { do }}$

if $A, B \in R(\Delta)$ then

Construct $\operatorname{NFR}(A \mid B)$;

Replace the summand $a(A \mid B)$ with $a N$ in $\Delta^{\prime}$, where $N$ is the leading

variable of $\operatorname{NFR}(A \mid B)$;

$\Delta^{\prime}:=\Delta^{\prime} \cup \operatorname{NFR}(A \mid B) ;$

if $(A \in R(\Delta)$ and $B \notin R(\Delta))$ or $(A \notin R(\Delta)$ and $B \in R(\Delta))$ then return NO;

if $A, B \notin R(\Delta)$ then

if there exists $Z \in S(\Delta)$ such that $A \mid B \sim Z^{|A| B \mid}$

then Replace the summand $a(A \mid B)$ with $a\left(Z^{|A| B \mid}\right)$ in $\Delta^{\prime}$;

else return NO;

6. return YES and $\Delta^{\prime}$;

Figure 1: An algorithm which (constructively) decides the membership to $\mathrm{nBPA}_{\tau} \cap \mathrm{nBPP}_{\tau}$ for normed $\mathrm{BPP}_{\tau}$ processes. 
are no communications in BPP, the notion of dual action is no longer sensible- -hence the second step of our algorithm can be removed in case of normed BPP processes.

Now we provide an analogous algorithm for normed $\mathrm{BPA}_{\tau}$ processes. We start with some auxiliary definitions and lemmas.

Definition 11 (CL sets). Let $\Delta$ be a normed BPA $A_{\tau}$ process in $G N F$. For each $Y \in S(\Delta)$ we define the set $C L(Y)$, composed of all $X \in \operatorname{Var}(\Delta)$ which fulfil the following conditions:

- If a $\alpha$ is a summand in the def. equation for $X$ such that Length $(\alpha) \geq 1$, then $\alpha \sim$ $Y^{|\alpha|-1} \cdot X$.

- The def. equation for $Y$ contains a summand bisimilar to a $Y^{k}, k \in N \cup\{0\}$, iff one of the following conditions holds:

1. $\alpha=\epsilon$ and the def. equation for $X$ contains a summand $a$

2. $\alpha \neq \epsilon$ and the def. equation for $X$ contains a summand which is bisimilar to $a\left(Y^{k-1} \cdot X\right)$.

3. $a=\tau$ and the def. equation for $X$ contains two summands of the form $b \alpha_{1}, \bar{b} \alpha_{2}$ such that $k=$ Length $\left(\alpha_{1}\right)+$ Length $\left(\alpha_{2}\right)-1$.

It is easy to see that the set $C L(Y)$ can be constructed in polynomial time for each $Y \in$ $S(\Delta)$. The following lemma is due to D. Caucal (see [Cau88]):

Lemma 4. Let $\Delta, \Delta^{\prime}$ be normed $B P A_{\tau}$ processes in $G N F$ and let $\alpha, \beta \in \operatorname{Var}(\Delta), \alpha^{\prime}, \beta^{\prime} \in$ $\operatorname{Var}\left(\Delta^{\prime}\right)$ such that $\beta \sim \beta^{\prime}$ and $\alpha . \beta \sim \alpha^{\prime}$. $\beta^{\prime}$. Then $\alpha \sim \alpha^{\prime}$

Lemma 5. Let $\Delta, \Delta^{\prime}$ be normed $B P A_{\tau}$ processes. Let $A_{1}, \ldots, A_{k} \in \operatorname{Var}(\Delta), X, Y \in$ $\operatorname{Var}\left(\Delta^{\prime}\right)$ such that $|X|=|Y|=1$ and $A_{1} \cdot \cdots . A_{k} \sim Y^{l} . X$ where $l=\left|A_{1} \cdot \cdots \cdot A_{k}\right|-1$. Then $A_{k} \sim Y^{\left|A_{k}\right|-1}$.X and $A_{i} \sim Y^{\left|A_{i}\right|}$ for $1 \leq i<k$.

Proof: Clearly $A_{k} \sim Y^{\left|A_{k}\right|-1} . X$. Hence $A_{1} \cdot \cdots \cdot A_{k-1} \sim Y^{\left|A_{1} \cdots \cdot A_{k-1}\right|}$ (due to Lemma 4). The fact $A_{i} \sim Y^{\left|A_{i}\right|}$ for $1 \leq i<k$ can be proved by induction on $k$. If $k=2$ then $A_{1} \sim Y^{\left|A_{1}\right|}$ and our lemma holds. If $k>2$, then clearly $A_{k-1} \sim Y^{\left|A_{k-1}\right|}$ and due to Lemma 4 we have $A_{1} \cdots \cdot A_{k-2} \sim Y^{\left|A_{1} \cdots \cdot A_{k-2}\right|}$. Now we can use induction hypothesis and conclude that $A_{i} \sim Y^{\left|A_{i}\right|}$ for $1 \leq i<(k-2)$.

Lemma 6. Let $\Delta$ be a normed reduced $B P A_{\tau}$ process in 3-GNF, $\Delta \in n B P A_{\tau} \cap n B P P_{\tau}$. Let $Q . \alpha$ be a reachable state of $\Delta$ such that $Q \in G(\Delta), \alpha \neq \epsilon$. Then there are unique variables $Y \in S(\Delta), X \in C L(Y)$ such that $Q . \alpha \sim Y^{|Q . \alpha|-1} . X$.

Proof: As $\Delta \in \mathrm{nBPA}_{\tau} \cap \mathrm{nBPP}_{\tau}$, there is a normed $\mathrm{BPA}_{\tau}$ process $\Delta^{\prime}$ in $\mathrm{INF}_{\mathrm{BPA}}$ such that $\Delta \sim \Delta^{\prime}$. Let $n=\max \left\{|A|, A \in \operatorname{Var}\left(\Delta^{\prime}\right)\right\}$. As $Q$ is growing, $Q \rightarrow^{*} Q \cdot \gamma$ where $\gamma \neq \epsilon$. Hence the state $Q \cdot \gamma^{n} \cdot \alpha$ is a reachable state of $\Delta$ and therefore there is a reachable state $\delta$ of $\Delta^{\prime}$ such that $Q \cdot \gamma^{n} \cdot \alpha \sim \delta$. As $\left|Q \cdot \gamma^{n} \cdot \alpha\right|>n$, we can conclude $\delta=R^{\left|Q \cdot \gamma^{n} \cdot \alpha\right|-1} . S$, where $R$ is a C-closure of $S$ (see Definition 8). Hence $Q \cdot \gamma^{n} \cdot \alpha \sim R^{\left|Q \cdot \gamma^{n} \cdot \alpha\right|-1} \cdot S$ and due to Lemma 5 we have $\alpha \sim R^{|\alpha|-1} . S$ and $Q \sim R^{|Q|}$, thus $Q . \alpha \sim R^{|Q . \alpha|-1} . S$. Now it suffices to show 
that there are $Y \in S(\Delta), X \in C L(Y)$ such that $Y \sim R$ and $X \sim S$. As $\Delta$ is normed, $Q \stackrel{s}{\rightarrow} * Y$ where $|Y|=1$ and $s$ is a norm-decreasing sequence of actions. Then $Q . \alpha \stackrel{s}{\rightarrow}$ * $Y . \alpha$ and as $Q . \alpha \sim R^{|Q . \alpha|-1} . S$, the state $R^{|Q . \alpha|-1} . S$ must be able to match the sequence $s$ and enter a state bisimilar to $Y . \alpha$. As $s$ is norm-decreasing and $|R|=1$, the only such state is $R^{|Y . \alpha|-1}$.S. Hence $Y . \alpha \sim R^{|Y . \alpha|-1} . S$ and due to Lemma 5 we have $Y \sim R$. The fact $Y \in S(\Delta)$ follows directly from Definition 8. As $S$ is a reachable state of $\Delta^{\prime}$, there is a variable $X \in S(\Delta)$ such that $X \sim S$. Clearly $X \in C L(Y)$ (see Definition 8). Variables $X, Y$ are unique because $\Delta$ is reduced.

It is worth noting that the variables $X, Y$ of the previous lemma need not be different-if a normed $\mathrm{BPA}_{\tau}$ process $\Delta$ belongs to $\mathrm{nBPA}_{\tau} \cap \mathrm{nBPP}_{\tau}$, then each $Y \in S(\Delta)$ belongs to $C L(Y)$.

To prove the corectness of our algorithm for normed $\mathrm{BPA}_{\tau}$ processes we need some lemmas about summands:

Lemma 7. Let $\Delta$ be a normed reduced $B P A_{\tau}$ process in 3-GNF and let $a(A . B)$ be a summand of a defining equation from $\Delta$ such that $A$ is non-regular and $B$ is regular. Then $\Delta \notin n B P A_{\tau} \cap n B P P_{\tau}$.

Proof: As $a(A . B)$ is a summand of a defining equation from $\Delta$ and $\Delta$ is normed and reduced, there is a reachable state of the form A.B. $\beta$. As $A$ is non-regular, $A \rightarrow^{*} Q . \alpha$ where $Q \in G(\Delta)$. Hence $Q . \alpha . B . \beta$ is a reachable state of $\Delta$ and due to Lemma 6 we have

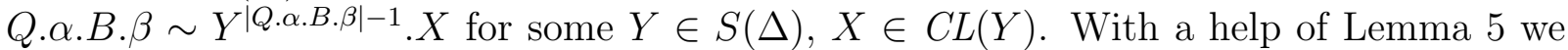
obtain $B \sim Y^{|B|}$ or $B \sim Y^{|B|-1}$. $X$ (the latter possibility holds if $\beta=\epsilon$ ). As $X, Y$ are growing, it contradicts regularity of $B$.

Lemma 8. Let $\Delta$ be a normed reduced $B P A_{\tau}$ process in 3-GNF. Let a $(A . B)$ be a summand of a defining equation from $\Delta$ such that $A$ is regular and $B$ is non-regular. Then it is possible to replace the summand $a(A . B)$ with aN where $N \notin \operatorname{Var}(\Delta)$ and to add a finite number of new equations in $I N F_{B P A}$ to $\Delta$ such that the resulting process $\Delta_{1}$ is bisimilar to $\Delta$.

Proof: As $A$ is regular, it is possible to construct $\Delta_{A}:=\operatorname{NFR}(A)$ such that $\operatorname{Var}(\Delta) \cap$ $\operatorname{Var}\left(\Delta_{A}\right)=\emptyset$. Now we modify defining equations of $\Delta_{A}$ slightly - each summand of the form $a$ where $a \in A c t$ is replaced with $a B$. The resulting system of equations is in $\mathrm{INF}_{\mathrm{BPA}}$. If we add the modified system $\Delta_{A}$ to $\Delta$ and replace the summand $a(A . B)$ with $a N$ where $N$ is the leading variable of $\Delta_{A}$, we obtain a process $\Delta_{1}$ which is clearly bisimilar to $\Delta$.

Lemma 9. Let $\Delta$ be a normed reduced $B P A_{\tau}$ process in 3-GNF and let $a(A . B)$ be a summand of a defining equation from $\Delta$ such that $A$ and $B$ are non-regular. Then

1. If $\Delta \in n B P A_{\tau} \cap n B P P_{\tau}$ then there are unique variables $Y \in S(\Delta), X \in C L(Y)$ such that $B \sim Y^{|B|-1} . X$

2. Let $B \sim Y^{|B|-1}$. $X$ for some $Y \in S(\Delta)$ and $X \in C L(Y)$. If there is a sequence of transitions $A=A_{0} \stackrel{a_{0}}{\rightarrow} A_{1} . \alpha_{1} \stackrel{a_{1}}{\rightarrow} A_{2} . \alpha_{2} \stackrel{a_{2}}{\rightarrow} \cdots \stackrel{a_{k}}{\rightarrow} A_{k} . \alpha_{k}$ such that $k \geq 0, A_{k} \in G(\Delta)$ and $A_{k} . \alpha_{k} \not Y^{\left|A_{k} \cdot \alpha_{k}\right|}$, then $\Delta \notin n B P A_{\tau} \cap n B P P_{\tau}$. 
3. Let $B \sim Y^{|B|-1}$. $X$ for some $Y \in S(\Delta)$ and $X \in C L(Y)$. If for each sequence of transitions $A=A_{0} \stackrel{a_{0}}{\rightarrow} A_{1} \cdot \alpha_{1} \stackrel{a_{1}}{\rightarrow} A_{2} \cdot \alpha_{2} \stackrel{a_{2}}{\rightarrow} \cdots \stackrel{a_{k}}{\rightarrow} A_{k} \cdot \alpha_{k}$ such that $A_{k} \in G(\Delta)$ the state $A_{k} . \alpha_{k}$ is bisimilar to $Y^{\left|A_{k} \cdot \alpha_{k}\right|}$, then the summand $a(A . B)$ can be replaced with $a N$ where $N \notin \operatorname{Var}(\Delta)$ and a finite number of new equations in $I N F_{B P A}$ can be added to $\Delta$ such that the resulting process $\Delta_{2}$ is bisimilar to $\Delta$.

\section{Proof:}

1. As $A$ is non-regular, $A \rightarrow^{*} Q . \alpha$ where $Q \in G(\Delta)$. The proof can be easily completed with a help of Lemma 5 and Lemma 6.

2. This is a consequence of Lemma 5 and Lemma 6 .

3. It suffices to realize that if $A=A_{0} \stackrel{a_{0}}{\rightarrow} A_{1} \cdot \alpha_{1} \stackrel{a_{1}}{\rightarrow} A_{2} \cdot \alpha_{2} \stackrel{a_{2}}{\rightarrow} \ldots \stackrel{a_{k}}{\rightarrow} A_{k} \cdot \alpha_{k}$ is a sequence of transitions such that $A_{0}, \ldots, A_{k-1} \notin G(\Delta)$ and $A_{k} \in G(\Delta)$, then Length $\left(A_{i} . \alpha_{i}\right) \leq$ $\operatorname{card}(\operatorname{Var}(\Delta))$ for $0 \leq i \leq k-1$ (here we use the assumption that $\Delta$ is in 3-GNF. Naturally, Length $\left(A_{i} \cdot \alpha_{i}\right)$ is bounded also in case of general GNF). As there are only finitely many sequences of variables of this bounded length, we can introduce a fresh variable for each of them. To construct the process $\Delta_{2}$, we use a similar procedure as in the proof of Lemma 8.

An existence of a sequence $A=A_{0} \stackrel{a_{0}}{\rightarrow} A_{1} \cdot \alpha_{1} \stackrel{a_{1}}{\rightarrow} A_{2} \cdot \alpha_{2} \stackrel{a_{2}}{\rightarrow} \ldots \stackrel{a_{k}}{\rightarrow} A_{k} \cdot \alpha_{k}$ such that $A_{k} \in G(\Delta)$ and $A_{k} . \alpha_{k} \nsim Y^{\left|A_{k}, \alpha_{k}\right|}$ is decidable in polynomial time:

Lemma 10. Let $\Delta$ be a normed reduced BPA process in 3-GNF. Let $A \in \operatorname{Var}(\Delta)$ be a non-regular variable and let $Y \in S(\Delta)$. The problem whether $A$ can reach a state of the form $Q . \alpha$ where $Q \in G(\Delta)$ and $Q . \alpha \nsim Y^{|Q . \alpha|}$ is decidable in polynomial time.

Proof: We divide the set $\operatorname{Var}(\Delta)$ into two disjoint subsets of successful and unsuccessful variables. $P \in \operatorname{Var}(\Delta)$ is unsuccessful if one of the following conditions holds:

- $P$ is growing and $P \not Y^{|P|}$.

- The defining equation for $P$ in $\Delta$ contains a summand of the form $a(R . S)$ where $R$ is non-regular and $S \nsim Y^{|S|}$.

A variable is successful if it is not unsuccessful. Furthermore, we define the binary relation ' $\Rightarrow$ ' on $\operatorname{Var}(\Delta): U \Rightarrow V$ iff $U$ is successful and the defining equation for $U$ in $\Delta$ contains a summand which is of one of the following forms:

- $a V$

- $a(V . W)$ where $W \in \operatorname{Var}(\Delta)$

- $a(W . V)$ where $W \in \operatorname{Var}(\Delta)$ is regular

Let ' $\Rightarrow{ }^{*}$ ' be the reflexive and transitive closure of ' $\Rightarrow$ '. It is not hard to prove that $A$ can reach a state of the form $Q . \alpha$ where $Q$ is growing and $Q . \alpha \neq Y^{|Q . \alpha|}$ iff $A \Rightarrow^{*} T$ for some unsuccessful variable $T$. As the relation ' $\Rightarrow{ }^{*}$ ' can be constructed in polynomial time, the proof is finished. 
Algorithm: A constructive test of the membership to $\mathrm{BBPA}_{\tau} \cap \mathrm{nBPP}_{\tau}$ for normed $\mathrm{BPA}_{\tau}$ processes.

Input: $\quad$ A normed reduced $\mathrm{BPA}_{\tau}$ process $\Delta$ in $3-\mathrm{GNF}$.

Output: $\quad$ YES and a normed $\mathrm{BPA}_{\tau}$ process $\Delta^{\prime}$ in $\mathrm{INF}_{\mathrm{BPA}}$ such that $\Delta \sim \Delta^{\prime}$ if $\Delta \in \mathrm{nBPA}_{\tau} \cap \mathrm{nBPP}_{\tau}$.

NO otherwise.

1. Construct the sets $S(\Delta), R(\Delta), G(\Delta)$ and for each $Y \in S(\Delta)$ construct the set $C L(Y)$.

2. If $(G(\Delta)=\emptyset)$ then

$\Delta^{\prime}:=\operatorname{NFR}(\Delta)$;

return YES and $\Delta^{\prime}$;

3. $\Delta^{\prime}:=\Delta$

4. for each summand of the form $a(A . B)$ in defining equations of $\Delta \underline{\text { do }}$

if $A, B \in R(\Delta) \underline{\text { then }}$

Construct $\operatorname{NFR}(A . B)$;

Replace the summand $a(A . B)$ with $a N$ in $\Delta^{\prime}$, where $N$ is the leading variable of $\operatorname{NFR}(A . B)$;

$\Delta^{\prime}:=\Delta^{\prime} \cup \operatorname{NFR}(A . B) ;$

if $A \notin R(\Delta)$ and $B \in R(\Delta) \underline{\text { then }}$ return NO;

if $A \in R(\Delta)$ and $B \notin R(\Delta)$ then

Construct the process $\Delta_{1}$ of Lemma 8 ;

$\Delta^{\prime}:=\Delta_{1} ;$

if $A, B \notin R(\Delta)$ then

if there exist $Y \in S(\Delta), X \in C L(Y)$ such that $B \sim Y^{|B|-1} . X$

then if $A$ can reach a state $Q . \alpha$ where $Q \in G(\Delta)$ and $Q . \alpha \neq Y^{|Q . \alpha|}$

then return NO;

else Construct the process $\Delta_{2}$ of Lemma 9 ;

$\Delta^{\prime}:=\Delta_{2}$

$\underline{\text { else return }}$ NO;

5. return YES and $\Delta^{\prime}$;

Figure 2: An algorithm which (constructively) decides the membership to $\mathrm{nBPA}_{\tau} \cap \mathrm{nBPP}_{\tau}$ for normed $\mathrm{BPA}_{\tau}$ processes. 
An algorithm which decides the membership to $\mathrm{nBPA}_{\tau} \cap \mathrm{nBPP}_{\tau}$ for normed $\mathrm{BPA}_{\tau}$ processes is presented on Figure 2. We use the same notation as in the case of $\mathrm{BPP}_{\tau}$.

There is a little shortcoming in the constructive variant of our algorithm for normed $\mathrm{BPA}_{\tau}$ processes - as the process $\Delta_{2}$ of Lemma 9 need not be in 3-GNF, the process $\Delta^{\prime}$ need not remain in 3-GNF either. But each lemma about summands (Lemma 7, 8, 9) is formulated for normed $\mathrm{BPA}_{\tau}$ process in 3-GNF. Naturally, it is not a problem to prove analogous lemmas about processes in general GNF - but we think that readability is more important feature than technical accuracy (note there is a similar problem in the constructive variant of our algorithm for normed $\mathrm{BPP}_{\tau}$ processes).

In case of normed BPA processes our algorithm must be slightly modified (and simplified). This is a consequence of the fact that a normed BPA process $\Delta$ belongs to $\mathrm{nBPA} \cap \mathrm{nBPP}$ iff it can be represented in INF - and INF is a little diffrent from $\mathrm{INF}_{\mathrm{BPA}}$ (see Definitions 9 and 8). Lemma 7 and Lemma 8 are valid also for normed BPA processes. Instead of Lemma 9 we can prove the following (in a similar way):

Lemma 11. Let $\Delta$ be a normed reduced BPA process in 3-GNF and let $a(A . B)$ be a summand of a defining equation from $\Delta$ such that $A$ and $B$ are non-regular. Then

1. If $\Delta \in n B P A \cap n B P P$ then there is a unique variable $Z \in S(\Delta)$ such that $B \sim Z^{|B|}$

2. Let $B \sim Z^{|B|}$ for some $Z \in S(\Delta)$. If there is a sequence of transitions $A=A_{0} \stackrel{a_{0}}{\rightarrow}$ $A_{1} \cdot \alpha_{1} \stackrel{a_{1}}{\rightarrow} A_{2} \cdot \alpha_{2} \stackrel{a_{2}}{\rightarrow} \ldots \stackrel{a_{k}}{\rightarrow} A_{k} \cdot \alpha_{k}$ such that $k \geq 0, A_{k} \in G(\Delta)$ and $A_{k} \cdot \alpha_{k} \not Z^{\left|A_{k} \cdot \alpha_{k}\right|}$, then $\triangle \notin n B P A \cap n B P P$.

3. Let $B \sim Z^{|B|}$ for some $Z \in S(\Delta)$. If for each sequence of transitions $A=A_{0} \stackrel{a_{0}}{\rightarrow}$ $A_{1} \cdot \alpha_{1} \stackrel{a_{1}}{\rightarrow} A_{2} \cdot \alpha_{2} \stackrel{a_{2}}{\rightarrow} \ldots \stackrel{a_{k}}{\rightarrow} A_{k} \cdot \alpha_{k}$ such that $A_{k} \in G(\Delta)$ the state $A_{k} \cdot \alpha_{k}$ is bisimilar to $Z^{\left|A_{k} \cdot \alpha_{k}\right|}$, then the summand $a(A . B)$ can be replaced with aN where $N \notin \operatorname{Var}(\Delta)$ and a finite number of new equations in INF can be added to $\Delta$ such that the resulting process $\Delta_{2}$ is bisimilar to $\Delta$.

Our algoritm for normed BPA processes differs from the algorithm on Figure 2 in two things - the sets $C L(Y)$ for $Y \in S(\Delta)$ are not computed at all and the last if statement in the loop of step 4 is replaced with the following code:

if $A, B \notin R(\Delta)$ then

if there exist $Z \in S(\Delta)$ such that $B \sim Z^{|B|}$

then if $A$ can reach a state $Q . \alpha$ where $Q \in G(\Delta)$ and $Q . \alpha \not Z^{|Q . \alpha|}$

then return NO;

else Construct the process $\Delta_{2}$ of Lemma 11 ;

$$
\Delta^{\prime}:=\Delta_{2}
$$

$\underline{\text { else return }}$ NO;

The existence of constructive variants of presented algorithms allow us to prove the following theorem:

Theorem 4. Bisimilarity is decidable in the union of normed $B P A_{\tau}$ and normed $B P P_{\tau}$ processes. 
Proof: Given two normed $\mathrm{BPA}_{\tau}$ or $\mathrm{BPP}_{\tau}$ processes, it is possible to check bisimilarity using algorithms which were published e.g. in [HJM94a] and [HJM94b]. If we get a normed $\mathrm{BPP}_{\tau}$ process $\Delta_{1}$ and a normed $\mathrm{BPA}_{\tau}$ process $\Delta_{2}$, then we run one of the constructive algorithms presented earlier. We can choose e.g. the first algorithm with $\Delta_{1}$ on input. If it answers NO, then $\Delta_{1} \not \Delta_{2}$. Otherwise we obtain a normed $\mathrm{BPP}_{\tau}$ process $\Delta_{1}^{\prime}$ in $\mathrm{INF}_{\mathrm{BPP}}$ which is bisimilar to $\Delta_{1}$. Now it suffices to check bisimilarity between two normed $\mathrm{BPA}_{\tau}$ processes $\overline{\Delta_{1}^{\prime}}$ and $\Delta_{2}$, where $\overline{\Delta_{1}^{\prime}}$ is obtained by running the algorithm presented in the proof of Proposition 3 with $\Delta_{1}^{\prime}$ on input.

Note that the corresponding statement holds for normed BPA and BPP processes by specialisation.

\section{Conclusions, Related Works and Future Research}

We have studied the class $\mathrm{nBPA}_{\tau} \cap \mathrm{nBPP}_{\tau}$ of those transition systems which can be generated by both $\mathrm{nBPA}_{\tau}$ and $\mathrm{nBPP}_{\tau}$ processes, i.e. the class of normed transition systems which can be equivalently (up to bisimilarity) expressed within the syntax of $\mathrm{BPA}_{\tau}$ and $\mathrm{BPP}_{\tau}$. We have shown that the problems whether a given $\mathrm{nBPA}_{\tau}$ or $\mathrm{nBPP}_{\tau}$ process $\Delta$ belongs to $\mathrm{nBPA}_{\tau} \cap \mathrm{nBPP}_{\tau}$ are decidable in polynomial time. We also provided constructive variants of these algorithms which are unfortunately not polynomial. Hence our algorithms for deciding bisimilarity in the union of $\mathrm{nBPA}_{\tau}$ and $\mathrm{nBPP}_{\tau}$ processes are not polynomial either. A simplified characterisation of $\mathrm{nBPA} \cap \mathrm{nBPP}$ and the corresponding algorithms are given as well. An obvious question is, whether there exists a decision algorithm of polynomial complexity. Another problem is, whether our results can be extended to the unnormed cases.

The problem whether a given nBPP process belongs to $\mathrm{nBPA} \cap \mathrm{nBPP}$ has been independently examined by Blanco in [Bla95] where it is shown that given a nBPP process, one can decide whether it is a nBPA process. His approach is based on well-known specification of properties of BPA transition graphs ([CM90]). A test whether a given nBPP graph fits this specification is given in the work. Consequently, his result does not allow for testing whether a given nBPA process belongs to the intersection as well as for generalisation for '-' operator. Also we would like to mention that our result on the classification of $\mathrm{nBPA} \cap \mathrm{nBPP}$ can be considered as a refinement of the result achieved in [Sch92] on the context-freeness of languages generated by Petri nets, as BPP processes form a proper subclass of Petri nets.

Our result about the classification of $\mathrm{nBPA}_{\tau} \cap \mathrm{nBPP}_{\tau}$ might be of some interest from the point of view of formal languages/automata theory as well. INF (for nBPA processes) can be taken as a special type of $\mathrm{CF}$ grammars which generate languages of the form $R .\left(L_{1} \cup \ldots \cup L_{n}\right)$, where $R$ is regular and each $L_{i}$ can be generated by a CF grammar having just one nonterminal and rules of the form $Z \rightarrow a Z^{k}, k \geq 0$. Considering language equivalence only, it is obvious that languages of the mentioned type $R$. $\left(L_{1} \cup \ldots \cup L_{n}\right)$ can be recognized by nondeterministic one counter automata. Hence our result on the 
classification of $\mathrm{nBPA} \cap \mathrm{nBPP}$ can be considered as a refinement of the result achieved in [Sch92] on the context-freeness of languages generated by Petri nets, as BPP processes form a proper subclass of Petri nets.

We hope a part of our work can be considered as one of the steps towards a solution of the open problem whether bisimilarity is decidable for PA processes. Furthermore, we would like to examine deeper the relationship between classes of behaviours which are generated by different types of syntax (e.g. Petri nets and BPA) and provide similar results like in the case of $\mathrm{nBPA}_{\tau}$ and $\mathrm{nBPP}_{\tau}$ processes - i.e. to characterise the "semantical intersection" and design algorithms which can decide (constructively) the membership to this intersection for both types of syntax. Last but not least corresponding complexity results should be provided.

\section{References}

[BBK87] J.C.M. Baeten, J.A. Bergstra, and J.W. Klop. Decidability of bisimulation equivalence for processes generating context-free languages. In Proceedings of PARLE'87, volume 259 of LNCS, pages 93-114. Springer-Verlag, 1987.

[BK88] J.A. Bergstra and J.W. Klop. Process theory based on bisimulation semantics. In Advanced Topics in Artificial Intelligence, volume 345 of LNCS, pages 50122. Springer-Verlag, 1988.

[Bla95] J. Blanco. Normed BPP and BPA. In Proceedings of ACP'94, Workshops in Computing, pages 242-251. Springer-Verlag, 1995.

[Cau88] D. Caucal. Graphes canoniques de graphes algebriques. Rapport de Recherche 872, INRIA, 1988.

[CHM93] S. Christensen, Y. Hirshfeld, and F. Moller. Bisimulation is decidable for all basic parallel processes. In Proceedings of CONCUR'93, volume 715 of $L N C S$, pages 143-157. Springer-Verlag, 1993.

[Chr93] S. Christensen. Decidability and Decomposition in Process Algebras. PhD thesis, The University of Edinburgh, 1993.

[CHS92] S. Christensen, H. Hüttel, and C. Stirling. Bisimulation equivalence is decidable for all context-free processes. In Proceedings of CONCUR'92, volume 630 of LNCS, pages 138-147. Springer-Verlag, 1992.

[ČKK96] I. Černá, M. Křetínský, and A. Kučera. Comparing expressibility of normed BPA and normed BPP processes. Technical Report FIMU-RS-96-02, Faculty of Informatics, Masaryk University, 1996.

[CM90] D. Caucal and R. Monfort. On the transition graphs of automata and grammars. Rapport de Recherche 1318, INRIA, 1990. 
[Gro91] J.F. Groote. A short proof of the decidability of bisimulation for normed BPA processes. Information Processing Letters, 42:167-171, 1991.

[HJM94a] Y. Hirshfeld, M. Jerrum, and F. Moller. A polynomial algorithm for deciding bisimilarity of normed context-free processes. Technical report ECS-LFCS-94286, Department of Computer Science, University of Edinburgh, 1994.

[HJM94b] Y. Hirshfeld, M. Jerrum, and F. Moller. A polynomial algorithm for deciding bisimulation equivalence of normed basic parallel processes. Technical report ECS-LFCS-94-288, Department of Computer Science, University of Edinburgh, 1994.

[HS91] H. Hüttel and C. Stirling. Actions speak louder than words: Proving bisimilarity for context-free processes. In Proceedings of LICS'91, pages 376-386. IEEE Computer Society Press, 1991.

[Kuč95] A. Kučera. Deciding regularity in process algebras. BRICS Report Series RS95-52, Department of Computer Science, University of Aarhus, October 1995.

[Mil89] R. Milner. Communication and Concurrency. Prentice-Hall, 1989.

[MM94] S. Mauw and H. Mulder. Regularity of BPA-systems is decidable. In Proceedings of CONCUR'94, volume 836 of LNCS, pages 34-47. Springer-Verlag, 1994.

[Mol96] F. Moller. Infinite results. In Proceedings of CONCUR'96, volume 1119 of LNCS, pages 195-216. Springer-Verlag, 1996.

[Par81] D.M.R. Park. Concurrency and automata on infinite sequences. In Proceedings $5^{\text {th }}$ GI Conference, volume 104 of LNCS, pages 167-183. Springer-Verlag, 1981.

[Sch92] S.R. Schwer. The context-freeness of the languages associated with vector addition systems is decidable. Theoretical Computer Science, 98(2):199-247, 1992.

[Sti96] C. Stirling. Decidability of bisimilarity. In Proceedings of INFINITY'96, MIP9614, pages 30-31. University of Passau, 1996. 